\title{
Babesia and its hosts: adaptation to long-lasting interactions as a way to achieve efficient transmission
}

\author{
Alain Chauvin $^{1 *}$, Emmanuelle Moreau ${ }^{1},{\text { Sarah } \text { Bonnet }^{2} \text {, Olivier Plantard }}^{3}$, \\ Laurence MALANDRIN ${ }^{3}$ \\ ${ }^{1}$ École nationale vétérinaire, UMR 1300 BIOEPAR, ENVN, Atlanpôle - La Chantrerie, BP 40706, \\ F-44307 Nantes Cedex 03, France \\ 2 INRA, UMR BIPAR, ENVA, 7 avenue du Général de Gaulle, 94700 Maisons-Alfort Cedex, France \\ ${ }^{3}$ INRA, UMR 1300 BIOEPAR, ENVN, Atlanpôle - La Chantrerie, BP 40706, F-44307 Nantes Cedex 03, France
}

(Received 4 August 2008; accepted 16 April 2009)

\begin{abstract}
Babesia, the causal agent of babesiosis, are tick-borne apicomplexan protozoa. True babesiae (Babesia genus sensu stricto) are biologically characterized by direct development in erythrocytes and by transovarial transmission in the tick. A large number of true Babesia species have been described in various vertebrate and tick hosts. This review presents the genus then discusses specific adaptations of Babesia spp. to their hosts to achieve efficient transmission. The main adaptations lead to long-lasting interactions which result in the induction of two reservoirs: in the vertebrate host during low long-term parasitemia and throughout the life cycle of the tick host as a result of transovarial and transstadial transmission. The molecular bases of these adaptations in vertebrate hosts are partially known but few of the tick-host interaction mechanisms have been elucidated.
\end{abstract}

Babesia / transmission / persistence / vertebrate host / tick host

\section{Table of contents}

1. Introduction.

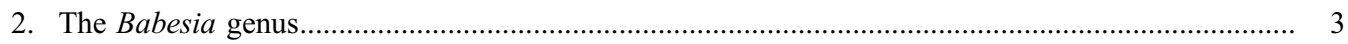

2.1. Phylogenetic position of Babesia spp. within the Apicomplexa............................................ 3

2.2. Specificities of the Babesia genus .................................................................................... 3

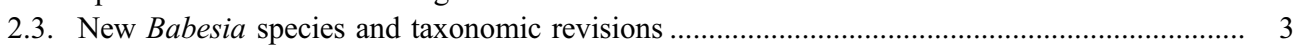

2.4. Babesia spp. life cycle ................................................................................................. 3

2.4.1. Development in vertebrate hosts ....................................................................... 3

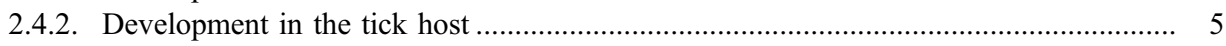

3. Transmission and adaptation of Babesia spp. to their hosts ........................................................ 5

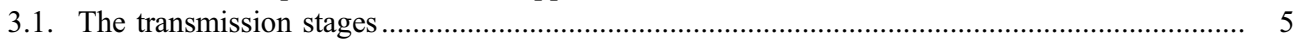

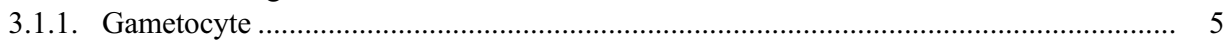

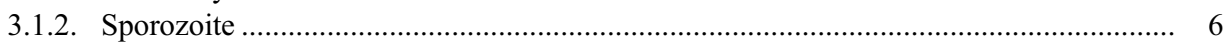

\footnotetext{
* Corresponding author: alain.chauvin@vet-nantes.fr
} 


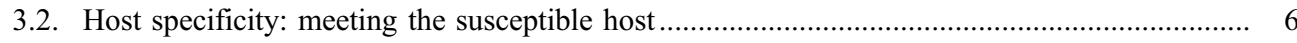

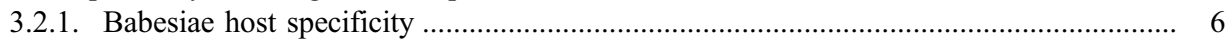

3.2.2. Tick host specificity .......................................................................................... 6

3.2.3. Influence of host specificities on transmission efficiency and parasite evolution ......... 6

3.3. Long-term persistence of babesiae in their hosts: transmission efficiency .............................. 7

3.3.1. Persistence in the vertebrate host ........................................................................ 7

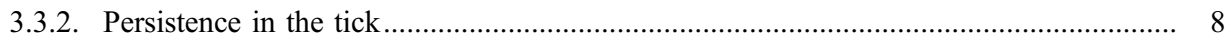

4. Molecular basis of babesiae adaptation to their hosts and thus to transmission ............................. 9

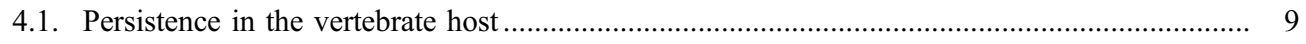

4.1.1. Immune response of the vertebrate host .................................................................. 9

4.1.2. Molecular and cellular escape strategies .................................................................. 9

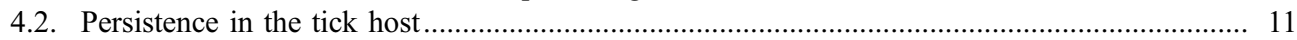

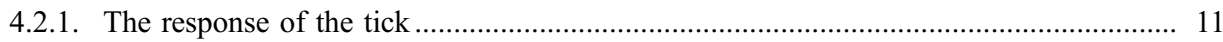

4.2.2. Adaptation of babesiae to transmission by ticks ........................................................ 12

4.3. Does babesiae use the vertebrate immune modulation induced by ticks to be

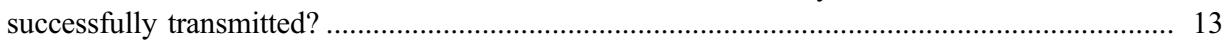

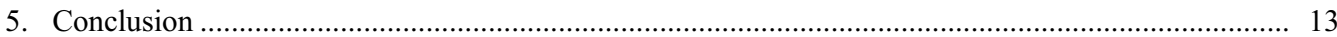

\section{INTRODUCTION}

Babesiosis is a worldwide tick-borne hemoprotozoosis affecting many mammalian species and caused by intraerythrocytic multiplication of apicomplexans in the Babesia genus. The evolutionary success of this parasite is attested by the large number of species described (more than 100 [62], with numerous species probably remaining to be discovered and/or described). It can be compared with the radiation of Plasmodium with which it shares phylogenetic proximity and numerous biological features. Both parasites are vector-borne protozoa transmitted by an arthropod, their life cycle including asexual multiplication in vertebrate blood cells, sexual reproduction in the vector and the production of sporozoites in the salivary glands of the vector. Babesia can thus be considered as a hemoprotozoan genus that is specifically adapted to using ticks as vectors (and not Diptera as in Plasmodium) [80].

Babesia and its two hosts, the tick vector and vertebrate host, represent a complex system in which the interactions between the three partners are among the longest described. First, the intimate contact between the tick and its host, i.e. the tick bite, lasts from 2 days to about 2 weeks, depending on the stage (larva, nymph or adult female) and species of the tick, thereby allowing an extended molecular dialogue between the tick and vertebrate host. In contrast, with mosquitoes or fleas, this contact lasts only a few seconds. After being introduced in a sporozoite form, mixed with tick salivary components, babesiae may then persist asymptomatically within its host for several years. Maintenance and persistence within the tick vector is ensured by transovarial and transstadial transmission sometimes over several tick generations, depending on the developmental duration and length of questing periods of the tick. All these features affect babesia transmission and are dependent on the adaptive strategies of all three partners.

This review starts with a brief presentation of the biology of babesiae, then focuses on the adaptive strategies shown by the Babesia genus in both vertebrate hosts and vectors. To date only a few genes are clearly known to be involved in babesia/tick or babesia/vertebrate interactions (unlike other vector-borne disease pathogens such as Plasmodium falciparum or Borrelia burgdorferi), so this review will concentrate on the specificities of the Babesia genus (notably its transovarian transmission) and their consequences on parasite transmission, evolution of the system and disease epidemiology. 


\section{THE BABESIA GENUS}

\subsection{Phylogenetic position of Babesia within the Apicomplexa}

The Babesia genus belongs to the Apicomplexa phylum, an early-branching eukaryotic lineage, characterized by the presence of an apical complex and a unique cytoskeleton distinct from that of other eukaryotes [49]. Although complete genomes of Apicomplexa belonging to different genera are already available (i.e. Plasmodium, Theileria, Babesia...), the phylogenetic relationships between the different vector-borne Apicomplexan groups are poorly documented [67]. This lack of knowledge hinders our understanding of the evolution of these parasites, which exploit both vertebrate blood cells and arthropod vectors. The key genes involved in particular in biological aspects of these parasites could be identified from comparison of genomes of closely related Apicomplexa with different biological features.

\subsection{Specificities of the Babesia genus}

Since the discovery of the genus at the end of the $19^{\text {th }}$ century, many different species associated with various domestic and wild animals have been described, and the tick species acting as a vector has been identified for the main Babesia species of domestic animals. Description and classification of Babesia spp. (and their distinction from the closely related Theileria) were initially based on morphological characteristics, notably the pattern of merozoite assembly in red blood cells. The two genera were defined according to the sites of multiplication in the vertebrate host and transmission modes within the tick host. Babesiae only multiply in the erythrocytes of the vertebrate host and are transovarially transmitted in the vector host. Thus, the Babesia genus clearly differs from Theileria which first multiplies in the lymphocytes and then the erythrocytes and is not transovarially transmitted within the tick. This restriction excludes ipso facto some species previously known as Babesia equi and Babesia microti from the Babesia genus. This restriction has been confirmed by molecular data and a monophyletic status has been conferred to Babesia spp. [6, 31-33, 107, 120] which develop in a wide range of vertebrate hosts (mostly mammals, but also birds [93]) and tick hosts (mostly hard ticks but also soft ticks [30]).

\subsection{New Babesia species and taxonomic revisions}

This progress in Babesia spp. identification, has led to the recognition of new cryptic/sibling species. For example B. canis, the babesia associated with the dog, was previously described as a single species. It has now been split into at least three distinct species or sub-species based on molecular and biological data. B. c. canis, B. c. rossi, B. c. vogeli have different tick hosts i.e. Dermacentor reticulatus, Haemaphysalis leachi and Rhipicephalus sanguineus respectively, leading to different epidemiological patterns $[12,26,120,126]$.

New Babesia species are also being isolated from either vertebrate or vector hosts. The former include some zoonotic Babesia (B. venatorum n. sp. i.e. Babesia sp. EU1 [57], B. sp. MO-1 [60] and the latter $B$. China BQ1 [54, 72]. Furthermore, many Babesia species were described long ago but biological data, notably identification of the vector, and molecular data are lacking for their formal characterization (reviewed by Penzhorn [94] and Uilenberg [120] for wild and domesticated animals respectively).

\subsection{Babesia spp. life cycle (Fig. 1)}

\subsubsection{Development in vertebrate hosts}

Vertebrate hosts are infected by the injection of sporozoites with saliva during the tick bite. In contrast to Plasmodium spp. or Theileria spp., Babesia spp. sporozoites penetrate directly into the red blood cells and all the parasitic stages develop in red blood cells. The parasite produces two merozoites by binary fission. After erythrocyte lysis, each merozoite invades a new erythrocyte and successive merogonies occur. Multiplication is asynchronous and various divisional stages of the parasite can be seen in the bloodstream at the same time. The size 


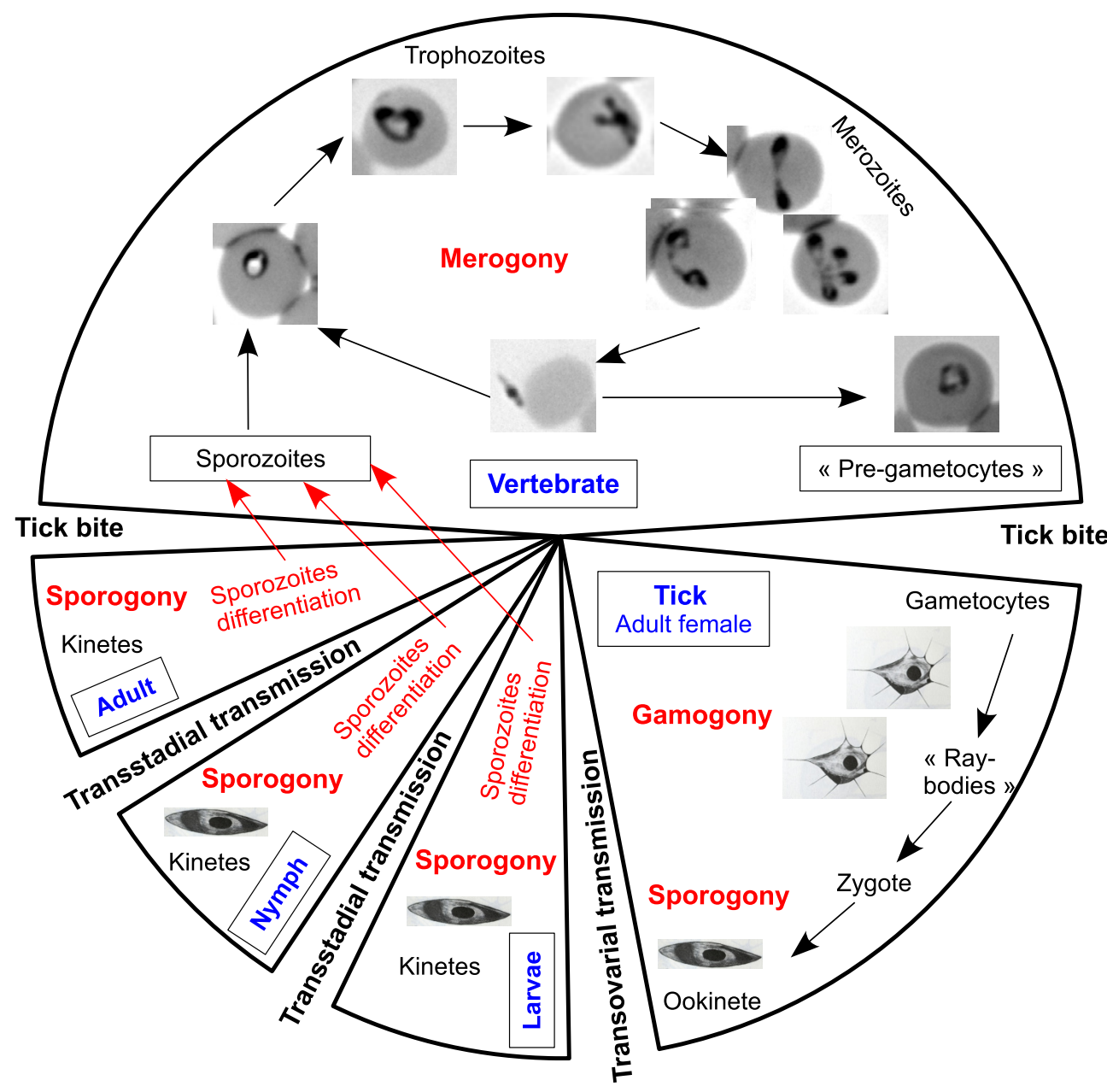

Figure 1. Babesia spp. life cycle. Merogonies in the vertebrate host are asynchronous and various divisional stages can be seen at the same time in the bloodstream: anaplasmoid forms (appearing just after penetrating the erythrocytes), rounded or ovoid forms (trophozoites), dividing forms, and merozoites, resulting from the binary fission, which are typically piriform and joined by their pointed extremities. Gamogony (gamete differentiation and zygote formation) occurs in tick intestinal cells. Ookinete and kinetes formed by asexual division of ookinete (sporogonies) occur in various tick organs. Final differentiation of sporozoites occurs in salivary glands. Graphic representation of kinetes and ray-bodies are line drawings reproduced from Sabine Wattendorff "dissertation zur Erlangang des Grades eines Doctor Medicinae Veterinariae", Hanover, 1980.

and location of the merozoites depend on both babesia and the host species. Babesia spp. are divided into two groups with (1) large babesiae (B. bigemina, B. canis, B. major, B. motasi...) in which the merozoites are longer than the erythrocyte radius and (2) small babesiae
(B. bovis, B. divergens, B. gibsoni, B. ovis...) in which the merozoites are smaller than the erythrocyte radius. This division into small and large babesiae, based on morphologic characteristics, has no clear genetic basis. Moreover, intraspecific phenotypic variability, with 
different parasite sizes or forms in bovine, gerbil or human blood cells, has been described for $B$. divergens [50].

\subsubsection{Development in the tick host}

When babesia-infected erythrocytes are ingested by ticks, most of the parasites degenerate and are destroyed. However, some specific stages of the parasite ("pre-gametocytes") survive and undergo further development (see review by Mehlhorn and Schein [81]) to evolve into gametocytes. These cannot be distinguished by light microscopy, but some differences are apparent under examination by transmission electron microscopy [103]. A few hours after ingestion, elongated bodies, with arrowheadshaped ray appear. These bodies, believed to be gamonts, are the so-called "strahlenkörper" ("ray bodies").

The gametes fuse in the lumen of the tick's digestive tract to form an elongated zygote 8 to $10 \mu \mathrm{m}$ in length bearing a spike-like arrowhead organelle, which facilitates cell penetration. Once across, the arrowhead touches the midgut cell membrane, which invaginates around this organelle at the point of contact. No parasitophorous membrane is produced and the midgut cell membrane appears to be lysed at the point of entry, apparently due to the action of enzyme released from a coiled structure in the invading parasite [82]. The function of the arrowhead appears to be very similar to that of rhoptries and micronemes in other protozoan parasites [104]. Once the babesia zygote has been internalized, the arrowhead organelle disintegrates and the zygote is then transformed into a motile stage, termed the ookinete. Meiosis, which indicates the beginning of sporogony in the Apicomplexa life cycle, probably occurs at this stage because the ookinete appears to be haploid [87]. The ookinete escapes from the midgut epithelium and invades the tick's body tissues. Invasion of the ovary in female ticks results in many babesia-infected eggs (transovarial transmission). Subsequent development of babesia includes asexual multiplication, continuing sporogony, and the development of numerous kinetes (sporokinetes). Sporogony takes place at each tick stage and the babesia infection acquired during one life stage is passed on to the next (transtadial transmission). Some kinetes also invade the salivary glands of ticks, where a final cycle of development produces the sporozoites. Sporozoites represent the infectious stage of the parasite as they introduce these protozoa into the mammalian host.

\section{TRANSMISSION AND ADAPTATION OF BABESIA SPP. TO THEIR HOSTS}

\subsection{The transmission stages}

A common feature of vector-transmitted Apicomplexan parasites is the existence of specific cellular stages involved in their transmission between vertebrate host and vector, namely the gametocyte (vertebrate to vector transmission) and the sporozoite (vector to vertebrate transmission). These stages are poorly characterized for Babesia species, and transmission studies (efficiency, rate, conditions of induction of transmissible stages, differential gene expression) are thwarted by the lack of knowledge of parasite development, the frequently insufficient amount of parasite material produced, and the absence of known specific markers.

\subsubsection{Gametocyte}

Babesia parasites share many characteristics with other Apicomplexa such as Plasmodium. One difference between the two hemoparasites is that babesiae do not produce completely differentiated gametocytes in the vertebrate. Five differentiation stages have been described in Plasmodium, the first being indistinguishable from the small trophozoites under light microscopy [115]. It is not known whether sexual differentiation in babesiae begins in the vertebrate host, with indistinguishable gametocytes, or only in the tick. Subsequent gametocyte differentiation is linked to the presence of induction factors in ticks and specific interactions between the two organisms. The sequential development of the tick-parasite stages was demonstrated when gut material from engorged 
female Rhipicephalus microplus was added to cultured B. bigemina, or when culture conditions (temperature, gas) were changed [51, 87]. Thus, temperature and some "factors" present in midgut extracts from engorged ticks, but not in the bovine erythrocytes used for culture, seem to be required. However, none of these factors has been identified to date.

One hypothesis is that the earlier gametocyte differentiation observed in Plasmodium spp. is specifically adapted to the life cycle of the dipteran vector in which only the adult stages are hematophagous. Plasmodium has to complete its entire lifecycle (sexual reproduction, production of sporozoites and migration to the salivary glands) rapidly within the adult stage of the vector in order to be inoculated into another vertebrate host. For Babesia spp. (and other tick-borne protozoa such as Theileria spp.), a single blood-meal is taken at each tick stage. The acquisition of transstadial transmission is a key adaptation of babesia to its vector.

\subsubsection{Sporozoite}

Kinetes are present in the salivary glands before or after tick attachment to its host. They differentiate within the salivary gland acini into polymorphic sporonts 60 to $300 \mu \mathrm{m}$ in diameter, with finger-like peripheric protrusions from which sporozoite formation is initiated. About five days after attachment, each large host cell may contain thousands of differentiated pyriform sporozoites with a broad apical pole showing organelles (rhoptries and micronemes) typical of the apical complex [81]. Similar developmental features have been described for B. occultans, B. ovata, B. gibsoni, B. ovis, $B$. bigemina, $B$. bovis and $B$. canis $[13,58$, $59,84,87,96,105]$. In the case of $B$. canis, no sporonts have been identified and the formation of typical pyriform sporozoites apparently resulted from successive binary fission of the kinetes [105].

Sporozoite differentiation only begins when the tick attaches to its vertebrate host. Temperature increase is involved in $B$. bovis and $B$. bigemina differentiation [37], but various factors may also be associated with the start of the blood meal, notably hypertrophy and increased metabolic and synthetic activities within the salivary glands.

\subsection{Host specificity: meeting the susceptible host}

\subsubsection{Babesiae host specificity}

With regards to the vector host, Babesia spp. specificity is usually restricted to a single tick species in a particular geographic area. Babesia spp. specificity for the vertebrate host was initially described as restricted. However, following the development of molecular tools, some Babesia species have been shown to have a wider vertebrate host range than previously thought. For example, B. bovis and B. bigemina, initially described as pathogens of cattle in tropical and sub-tropical areas, were both recently identified by specific serology and PCR in white-tailed deer (Odocoileus virginianus) in northern Mexico [24]. B. divergens is another parasite of cattle in temperate climates. However, it is also able to infect splenectomized humans [24], primates (chimpanzees and rhesus monkeys) [44], ungulates (roe deer, fallow deer, red deer, mouflon and sheep) [25, 40], and rodents (rat) [95] as well as non-splenectomized reindeer [88], sheep [77] and gerbils [69].

\subsubsection{Tick host specificity}

Two groups of ticks can be distinguished in relation to their feeding preference: most ticks, like Rhipicephalus (Boophilus) microplus, the vector of $B$. bigemina and $B$. bovis (which feed on cattle and other ruminants) are host-specific or specialist. A minority, which includes Ixodes ricinus, vector of $B$. divergens, $B$. venatorum and probably $B$. capreoli, is relatively unselective opportunistic or generalist parasites and utilize a wide range of vertebrates as hosts.

\subsubsection{Influence of host specificities on transmission efficiency and parasite evolution}

The probability of transmission of a Babesia spp. isolate to its specific vertebrate host is increased if the tick species shows restricted 
specificity. Subsequent evolution of the geographical distribution of the Babesia species will be highly correlated with tick species distribution. Evidence of modifications in tick-borne disease distributions has been demonstrated, in the context of global change [100], and explained by changes in tick distribution, tick population dynamics and tick-vertebrate host contact. Tick expansion could easily be followed by babesia expansion, as observed for $B$. canis canis, transmitted by $D$. reticulatus in Hungary [111]. In general, both reservoirs of the Babesia species, namely the tick and vertebrate host species, need to be present in the new habitat to allow Babesia species expansion.

In contrast, a wider host range for both tick and Babesia species, will facilitate transmission to new hosts, together with subsequent adaptation and potential evolution towards new species. Babesia spp. merozoites are able to invade erythrocytes from a wide range of animal species and sometimes replicate in vitro, $[42,127]$, thus providing possibilities for these parasites to adapt and develop to new hosts. For example, considerable diversity was observed in the ability of sheep erythrocytes to permit growth of $B$. divergens in vitro, ranging from refractoriness to full susceptibility. Experimental infection of sheep with refractory erythrocytes failed, whereas a long-term chronic infection (up to two years) was observed in vivo with fully susceptible erythrocytes [77].

Generalist ticks will be more efficient in presenting Babesia spp. isolates to "new" potential hosts. A striking feature of $B$. bovis, B. bigemina and $B$. divergens, which express less host specificity than most Babesia species, is the ubiquity of the transmitting vectors ( $R$. microplus and $R$. annulatus for both $B$. bovis and B. bigemina, and $I$. ricinus for $B$. divergens) which feed on an extremely wide range of vertebrate hosts. The genetic similarity of some ruminant Babesia species, such as $B$. divergens and $B$. capreoli carried by the same tick species, might also be due to their recent divergence.

If the length of the babesia life cycle is considered as the delay between two successive zygotes which are produced in the tick host, the probability of efficient sporozoite transmission will be influenced by the specificity of the tick. If this specificity is restricted, the babesia sporozoite will encounter a suitable host with each tick bite. If a babesia-infected generalist tick feeds on a non-receptive babesia-host, efficient sporozoite transmission will be delayed until the next stage or generation of the tick. This phenomenon will be amplified by tick biology and the generation time in monophasic ticks, which complete their life cycle in a few weeks, will be particularly short. In contrast, the generation time of babesia transmitted by a generalist tick, such as I. ricinus, which takes one to several years to complete its life cycle, will be up to 50 times longer. Since genetic recombination in babesia only occurs during sexual reproduction, these discrepancies in the life cycle length between Babesia species must be taken into account in any comparative analysis of gene evolution.

\subsection{Long-term persistence of babesiae in their hosts: transmission efficiency}

\subsubsection{Persistence in the vertebrate host}

\subsubsection{Primary infection}

The tick injection of babesia sporozoites and subsequent cycles of intraerythrocytic replication of the merozoites will induce a reaction from the host immune system. Even with the primary infection, the clinical expression of babesiosis is highly linked to the status of the individual animal (breed, age). Some breeds of dogs (Beagle, Fox terrier, Porcelain, Dachshund, mongrels) are more resistant to $B$. canis than others (Spaniel, Cocker spaniel, Griffon, Yorkshire terrier, Doberman, Pekinese) [79]. Three different phenotypes have been described in the Bos taurus/B. bovis system: "susceptible" animals with severe clinical signs which may lead to death, "intermediate" animals with mild clinical signs and "resistant" animals with no clinical signs [10]. These differential effects of babesia infection, depending on the species, breed or individual of the vertebrate host, demonstrate the genetic basis of resistance to babesiae.

An inverse relationship, between age and resistance to babesiae, has been reported with young animals being more resistant than adults. 
This phenomenon was observed in calves infected with $B$. divergens, $B$. bovis and $B$. bigemina [29, 66, 117] and in gerbils infected with $B$. divergens [65] but not in dogs infected with $B$. canis $[78,122]$. In the case of $B$. divergens, inverse age resistance only leads to differential clinical expression between young and old cattle but does not prevent the establishment of a persistent infection. The mechanisms underlying this inverse age resistance remain unknown but seem to be due to differences in localisation and timing of the immune mechanisms (reviewed by Zintl et al. [129]).

\subsubsection{Premune immunity}

Resistance to re-infection, acquired as a result of the continuous presence of the parasite, is known as "premune immunity" or premunity. During infection of cattle with $B$. bovis, $B$. bigemina or $B$. divergens $[5,36,61,75]$, of sheep (splenectomized or intact) with $B$. divergens $[28,77]$ or with $B$. ovis [55], and of dogs with B. canis or B. gibsoni $[15,16$, $78,122]$, the parasite can persist for several months, even several years, at a very low level of parasitemia. Sterilizing immunity has been demonstrated in dogs infected with $B$. canis, but only occurs in some individuals and asymptomatic carriers with premune immunity may be present in dog populations [16, 122]. Sterilizing immunity is also suspected for $B$. divergens infection in some bovines by Davies et al. [36].

\subsubsection{Advantages of long-lasting persistence in hosts}

Babesiae appear to be particularly well adapted to long-term persistence in the environment by using the vertebrate host as a reservoir. Acute development or parasite clearance from the blood stream could be interpreted as a sign of mal-adaptation between the Babesia species and its vertebrate hosts, as observed for gerbil infection with $B$. divergens [128]. Even in the natural well-adapted host, a delayed, unsuitable or insufficient immune response could lead to clinical manifestations of babesiosis and eventually to death of the infected host, which would then have a detrimental outcome on parasite transmission.
However, transmission to ticks at a low level of parasitemia must be the prevalent modality of babesia transmission in the field, given the longterm maintenance of the parasite in a biotope and generally asymptomatic carriage. The establishment of low and long-lasting parasitemia presents many advantages for babesia. Notably, it increases the duration of the transmission period and thus the chance of being acquired by the tick. In the case of $B$. bovis, the infection rates of the larval progeny did not differ significantly whether the females were engorged during acute or persistent infections of calves [61]. Furthermore, with $B$. ovis, low parasitemia has been observed to save the tick from deleterious effects, which occur when large numbers of parasites are acquired during the blood meal, [123] and thus indirectly improves transmission by preserving vector fitness.

\subsubsection{Persistence in the tick}

The transovarial and transstadial transmission exhibited by babesiae can be considered as a major adaptation. The fact that babesiae persist in all tick stages, means that both vertebrates and ticks serve as parasite reservoirs in the field thereby facilitating the long-term persistence of Babesia species in the ecosystem. In Plasmodium, the parasite is associated solely with the blood-feeding stage of the vector (i.e. adult insects) which may have several blood meals during this stage. In contrast, transstadial transmission can be considered as a specific adaptation to the tick life cycle, which involves only one blood meal per tick stage (i.e. larva, nymph and adult), by allowing parasite transmission during each of those stages. Transstadial transmission is observed in most tick-borne pathogens (Borrelia burgdorferi, Anaplasma phagocytophilum...), whereas transovarial transmission is more rarely observed in tick-transmitted pathogens and is a characteristic of the Babesia genus as compared with Theileria. It can be hypothesized that this transovarial transmission permits completion of the parasite's entire life cycle (longer than that of bacteria, notably due to sexual reproduction). In the field, when only the adult tick host feeds on a suitable babesia-host, 
transovarial transmission will increase transmission efficiency. It can also be considered as a major adaptation for long-lasting persistence, because some ticks remain infected and infective for several generations without needing to again feed on infected animals [39, 64, 125].

On account of this transovarial transmission, Babesia spp. can be considered as verticallytransmitted parasites. Theoretical considerations of virulence evolution [71] suggest that such parasites should evolve towards lower virulence to their hosts (both mammals and ticks in the present case), compared to a parasite with pure horizontal transmission. It would be interesting to compare the effects, on their vectors, of phylogenetically closely-related species that differ in their mode of transmission (e.g. Theileria and Babesia).

\section{MOLECULAR BASIS OF BABESIAE ADAPTATION TO THEIR HOSTS AND THUS TO TRANSMISSION}

\subsection{Persistence in the vertebrate host}

\subsubsection{Immune response of the vertebrate host}

Immune mechanisms are directed against erythrocytes infected with $B$. bovis or against free merozoïtes (Fig. 2). The role of the cellular response in protection against the parasite is demonstrated by the high susceptibility of splenectomized animals to babesia infection. Furthermore, immune responses led to the appearance of intracellular "crisis" forms [23, 53]. The development of these degenerating parasites seems to be caused more by the cellular immune responses than by the humoral immune responses [129]. During acute infection with $B$. bovis, the innate immune response seems to be essential and requires the production of interleukin-12 (IL12) and interleukin18 (IL18). These stimulate natural killer (NK) cells to produce high levels of gamma interferon (IFN $\gamma$ ) [43, 48, 108], which induce the production of nitric oxide (NO) by the macrophages [48]. During chronic infection, lysis of the infected red blood cells (iRBC) is also mainly mediated by the NO produced by sple- nic macrophages activated by IFN $\gamma$ and Tumor Necrosis Factor alpha (TNF $\alpha)$. This mechanism is then regulated by type 1 cytokines and is inhibited by interleukin-4 (IL4) and interleukin-10 (IL10) [46, 47]. The humoral immune response does not permit parasite clearance but several mechanisms help to control parasitemia [20]: antibody-dependent cellular cytotoxicity (ADCC) mediated by IgG1, described in vitro by Goff et al. [45], opsonization mediated by $\operatorname{IgG} 2$, neutralization of adherence of the free merozoïtes to RBC and of the cytoadherence of iRBC to endothelial cells [21], and activation of complement mediated by IgG1 and IgG2 [19].

The evolution of acute infection depends on the timing and location of production of the inflammatory and type 1 cytokines and on the quantities produced. A comparison of the immune response of calves and adult cattle against $B$. bovis showed that the innate response is only protective if the IFN $\gamma$ and IL12 are produced early on, before IL10 production. When IL10, IL12 and IFN $\gamma$ are produced at the same time, the type 1 response and the production of NO in the spleen are delayed or decreased, which allows disease expression [46]. On the contrary, during chronic infection, low level persistence of the parasite requires activation of the adaptive immune response: Th0 or Th1 lymphocytes activate the production of IgG, particularly the opsonizing IgG2 and iRBC lysis mechanisms mediated by macrophages and NO [20].

In $B$. ovis, $B$. divergens, $B$. canis or $B$. gibsoni only the humoral immune response has been studied, the production of antibodies beginning approximately 7 days after infection and persisting for several months $[16,55,90$, 116]. Antibodies do not seem to permit the clearance of $B$. bovis $[9,16,55,70]$ and antibody production is associated with asymptomatic carrying of $B$. divergens [76].

\subsubsection{Molecular and cellular escape strategies}

Babesia species have developed strategies to avoid or limit effects of the immune response which enable them to persist inside the vertebrate host, and thus to increase their likelihood of being transmitted. 


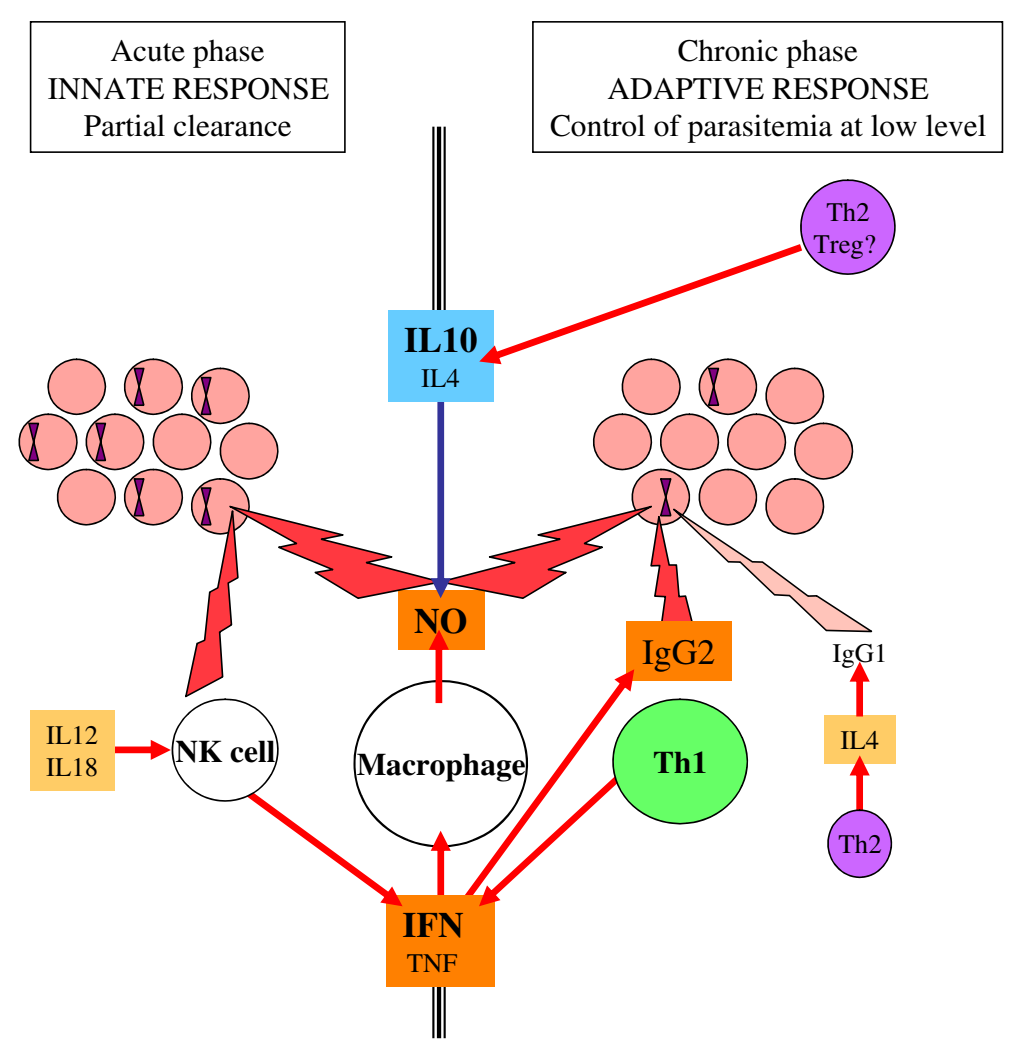

Figure 2. Effector immune mechanisms and their regulation developed by the vertebrate during $B$. bovis infection. NO secreted by IFN $\gamma$-activated macrophages is the major effector mechanism against iRBC. During the acute phase, the innate response regulated by inflammatory and type- 1 cytokines leads to partial elimination of the parasites injected by the tick. During the chronic phases, Th-1 cells and antibodies control parasitemia at a low level. IL4 and IL10 secreted by Th-2 or regulatory cells inhibit the activation of macrophages.

\subsubsection{Modulation of the immune response}

This persistence may result from a modulation of the host immune response. Lymphocyte blastogenesis and antibody production is depressed in dogs infected with $B$. gibsoni [1] and the specific immune response against $R$. microplus or Foot and mouth disease vaccine is decreased in B. bovis infected animals [22, 101]. B. bovis is also able to directly stimulate some components of the immune system, leading to the production of NO, IL12 and TNF $\alpha$ by macrophages [113] or the proliferation of lymphocyte B induced by the DNA and the CpG oligonucleotide [18, 109]. Although it might seem surprising that $B$. bovis stimulates an immune response which is finally fatal for it, some host immune mechanisms and parasite evasion mechanisms in fact help to develop the clinical expression of babesiosis. In $B$. bovis, these include the production of high levels of inflammatory cytokines, cytoadherence responsible for vascular disturbances (vasodilatation, low blood pressure, increased vascular permeability) and blood stasis in the microvessels. Highly pathogenic effects will be unfavorable to both babesia and its host. Similarly, the observed inverse age-related resistance in $B$. divergens infection is explained by Zintl et al. [129] as resulting from early NO production in the spleen of naïve young 
animals, which protects them from clinical disease. In contrast, naïve adults express a delayed systemic inflammatory response and are much more susceptible to disease. Infection can thus occur in young individuals of the vertebrate host without apparent damage.

\subsubsection{VESA: Antigenic variation and cytoadherence}

The ability of some Babesia species to adhere to endothelial cells, leading to sequestration of $\mathrm{iRBC}$ in the microvasculature, has been demonstrated $[35,89,106]$. This enables the babesia to persist within the host by preventing the passage of $\mathrm{iRBC}$ in the spleen and thus their clearance from the blood stream. This adhesive mechanism, by retaining the $\mathrm{iRBC}$ in the microvessels, could improve parasite transmission when the densest parasite population is close to the tick bite sites.

Cytoadherence in B. bovis is mediated by Variant Erythrocyte Surface Antigen 1 (VESA1), a heterodimeric protein exported to the surface of the infected host erythrocyte and implying prolonged contact with the host immune system. The parasite has thus developed a strategy to protect its adhesive function from immune interference, notably by preserving cytoadhesion even when antibodies are produced, in the form of antigenic variation [2, 4, 34]. VESA1 is encoded by the multigenic ves family [3], which includes about 150 ves genes distributed in clusters of two or more genes throughout all four $B$. bovis chromosomes [17]. Antigenic variation occurs through segmental gene conversion between areas of conserved stretches of nucleotide identity and allows rapid variation of the exposed epitopes within the initial parasite population.

\subsubsection{Antigenic polymorphism}

Babesia parasites cause disease when the host immune defenses are overcome by invasion and replication of merozoites within the host erythrocytes. Intraerythrocytic babesiae are partially protected from the host immune response, whereas free merozoites are fully exposed, if only for a short time. The merozoite surface is coated with glycosyl phosphatidyl anchored proteins that are involved in initial attachment to the host erythrocyte surface and are immunodominant targets for host-protective antibodies. They differ strikingly from one species to another, and also exhibit a high degree of intra-species antigenic polymorphism. These surface-exposed proteins have been studied mainly in $B$. divergens and B. bovis $[25,124]$. The extent of $B$. divergens surface-coating protein $\mathrm{Bd} 37$ polymorphism was evidenced by PCR-RFLP and five clades have been described [56]. Sequencing revealed an overall sequence identity ranging from 54 to $88 \%$ and different surface-exposed epitopes between isolates [97]. In B. bovis, Variable Merozoite Surface Antigen (VMSA) is a multigene family consisting of five members located at two different genomic loci: msa-1 and msa-2 [41, 114]. The overall sequence divergence for Merozoite Surface Antigen (MSA)-1 is the greatest, with identities ranging from 19 to $99 \%$, compared to 64 to $100 \%$ for MSA- $2 \mathrm{a} / \mathrm{b}$ and 85 to $100 \%$ for MSA-2c [11, 68]. Analysis of the MSA molecules of vaccine breakthrough isolates revealed extensive sequence variations depending on the vaccine strain used, which resulted in a complete lack of immunologic cross-reactivity for MSA-1 [68]. This surface polymorphism allows the parasite to evade the host immune system at the population level.

\subsection{Persistence in the tick host}

\subsubsection{The response of the tick}

Because Babesia spp. exploit several tick tissues during the life cycle stages spent within this vector (notably epithelium cells of the digestive tract, salivary glands, or oocytes, and also tick nutrients for their growth and multiplication), these parasites must have a negative effect on tick fitness [27, 38, 52, 91]. Although low parasitemia in the vertebrate host may limit the pathogenic effect of these protozoa [123], the induction of an adequate defense response against babesiae could actually be advantageous to them by preventing excessive effects on tick development and thus facilitating babesia transmission.

Ticks have probably evolved several defense mechanisms to control babesiae during their 
long-lasting and intimate interactions with these parasites. In the Plasmodium/diptera system, a genetic origin of refractoriness to Plasmodium (failure of ookinetes to develop into oocysts in mosquito midgut) has already been identified in Anopheles gambiae populations [83]. Although studies are in progress on the innate immunity of insects, little information is available for acari. This gap in our knowledge may rapidly be filled by recent developments in acarus genomics [92].

Two molecules, involved in tick defense against babesiae and theileiriae have been formally described in Haemaphysalis longicornis: longicine [118] and longipain [119].

Longicine is an antimicrobial peptide produced in the tick midgut epithelium, which suggests that it is secreted into the lumen. It was demonstrated in vitro, that longicine adheres specifically to the membrane of the Theileria equi merozoite, inhibits proliferation and kills parasites. In vivo, the peptide cleared B. microti infection in the mouse and RNA interference data demonstrated that endogenous longicine is able to directly kill $B$. gibsoni in $H$. longicornis. Anti-microbial peptides have been largely described in Ixodidae and I. ricinus defensin is strongly induced in the midgut after Borrelia infection [102]. This defense mechanism is likely to be involved in other babesia-tick systems.

Longipain is a cathepsin B protease. It belongs to a network of aspartic and cysteine proteases that are conserved in a wide range of parasites, including nematodes, flatworms and ticks and involved in blood meal digestion [110]. This cathepsin protease system differs from that of insects which are based on serine proteases. Longipain is expressed in the tick midgut epithelium during the blood meal and is possibly released into the lumen. Longipain is able to kill babesia merozoites in vitro by specific adherence to the parasite membranes, but this parasitic stage does not naturally develop in ticks. Even if there is no evidence of activity on babesia gametocytes, RNA interference data demonstrated that longipainknockdown $H$. longicornis showed a 3-fold increase in its ability to transmit $B$. gibsoni.

The expression of proteins in the midgut and ovaries of $R$. microplus, fed either on healthy or
B. bovis infected calves, were investigated and compared in two proteomic studies [98, 99]. A total of 16 and 19 proteins which are upor down-regulated during babesia infection have been described in the midgut and ovaries respectively. No data are available on the positive or negative effects of these molecules on babesia development but this protein database could be an important tool in further studies.

Of the different factors permitting parasite development in its vector (i.e. factors inducing sexual development, receptors and/or factors allowing crossing of the midgut, ovary or salivary gland barriers...), only one tick molecule has as yet been identified. The $H$. longicornis vitellogenin receptor is a $197 \mathrm{kDa}$ protein expressed on the oocyte plasma membrane during ovary development. RNA interference studies have demonstrated the critical role of this protein in transovarial transmission of $B$. gibsoni [8]. The lack of data on this subject is partly due to the difficulties encountered in experimental reproduction of the babesia life cycle. The recent artificial infection of ticks with babesia culture in vitro [14] as well as the use of experimental vector hosts, like soft ticks [7], may provide a powerful tool in future studies.

\subsubsection{Adaptation of babesiae to transmission by ticks}

The mechanisms of babesia development in the tick remain largely unknown. For example, true gametocytes have not been conclusively identified in the life cycle of any Babesia species and indirect evidence that sexual reproduction does occur has been obtained only for $B$. divergens [73], B. bigemina and B. canis [74]. Furthermore, our knowledge of babesia gene expression in the tick is extremely limited. The only study concerned B. bovis sporozoites, which are produced in huge numbers inside the salivary glands and thus are more easily purified from tick extracts. It is hypothesized that since sporozoites share the same target cell as merozoites, they would use the same proteins to invade the erythrocyte. The presence of MSA-1, MSA-2, and the Rhoptry-AssociatedProtein 1 (RAP-1) in sporozoites as well as 
their involvement in erythrocyte attachment and invasion were confirmed $[85,86]$.

\subsection{Does babesiae use the vertebrate immune modulation induced by ticks to be successfully transmitted?}

The modulation of host defense by tick saliva is known to facilitate the transmission of bacteria like Borrelia (see [117b]). Tick saliva contains several active components which facilitate the long feeding stage of the tick on the vertebrate host (reviewed by Steen et al. [112]). Functional assays in vitro suggest that some of these components may also interact with the anti-babesia response. Indeed, factors from Amblyomma americanum and $H$. longicornis that inhibit macrophage migration were also shown to inhibit the migration of human monocytes and might decrease macrophage infiltration of the tick bite lesion [63, 121]. Moreover, a $15 \mathrm{kDa}$ protein from Ixodes scapularis, exhibiting some sequence similarity with transforming growth factor, can modulate the pathway of IL2 transcription by T-cells and a serine protease inhibitor (Iris) from I. ricinus is able to decrease cytokine production by leukocytes. Further studies are needed to explore the role of the tick saliva in the establishment of babesia in the vertebrate host.

\section{CONCLUSION}

Adaptive strategies of Babesia spp. to transmission by ticks have led to long-lasting interactions between Babesia spp. and its hosts. Chronic low parasitemia in the vertebrate host and transovarial and transstadial transmission in the tick host results in a dynamic equilibrium in the babesia-host interactions which enables each partner to survive at the lowest possible cost. This ensures optimal fitness for the entire system and allows the long-term persistence of Babesia spp. in the ecosystem, since both its hosts serve as reservoirs. Some mechanisms leading to long-term maintenance in vertebrate hosts have been well described but only for a few Babesia species. Recent studies have explored tickpathogen interactions, but few have focused on babesia-tick interactions and none has examined babesia gene expression in its vector hosts. The evolution of new strains (or even species) of babesia will depend both on the tick vector meeting a new host and on the capacity of Babesia sp. to develop in this new host. These Babesia species will exhibit different selective patterns depending on the biological characteristics of the tick (length of the life cycle, specificity). A comparative analysis of gene evolution in Babesia spp. and its tick vector should provide a key to understanding babesia-host interactions in different ecosystems.

\section{REFERENCES}

[1] Adachi K., Ueno C., Makimura S., Immunosuppression in dogs naturally infected with Babesia gibsoni, J. Vet. Med. Sci. (1993) 55:503-505.

[2] Allred D.R., Cinque R.M., Lane T.J., Ahrens K.P., Antigenic variation of parasite-derived antigens on the surface of Babesia bovis-infected erythrocytes, Infect. Immun. (1994) 62:91-98.

[3] Allred D.R., Carlton J.M., Satcher R.L., Long J.A., Brown W.C., Patterson P.E., et al., The ves multigene family of $B$. bovis encodes components of rapid antigenic variation at the infected erythrocyte surface, Mol. Cell (2000) 5:153-162.

[4] Allred D.R., Molecular technology and antigenic variation among intraerythrocytic hemoparasites: do we see reality?, Vet. Parasitol. (2001) 101:261-274.

[5] Allred D.R., Al-Khedery B., Antigenic variation as an exploitable weakness of babesial parasites, Vet. Parasitol. (2006) 138:50-60.

[6] Allsopp M.T., Allsopp B.A., Molecular sequence evidence for the reclassification of some Babesia species, Ann. N.Y. Acad. Sci. (2006) 1081:509-517.

[7] Battsetseg B., Matsuo T., Xuan X., Boldbaatar D., Chee S.H., Umemiya R., et al., Babesia parasites develop and are transmitted by the non-vector soft tick Ornithodoros moubata (Acari: Argasidae), Parasitology (2007) 134:1-8.

[8] Boldbaatar D., Battsetseg B., Matsuo T., Hatta T., Umemiya-Shirafuji R., Xuan X., Fujisaki K., Tick vitellogenin receptor reveals critical role in oocyte development and transovarial transmission of Babesia parasite, Biochem. Cell Biol. (2008) 86:331-344.

[9] Ben Musa N., Dawoud H.A., The protective activity of serum and fractionated serum from rats against Babesia divergens, J. Egypt. Soc. Parasitol. (2004) 34:407-422. 
[10] Benavides M.V., Sacco A.M., Differential Bos taurus cattle response to Babesia bovis infection, Vet. Parasitol. (2007) 150:54-64.

[11] Berens S.J., Brayton K.A., Molloy J.B., Bock R.E., Lew A.E., McElwain T.F., Merozoite surface antigen 2 proteins of Babesia bovis vaccine breakthrough isolates contain a unique hypervariable region composed of degenerate repeats, Infect. Immun. (2005) 73:7180-7189.

[12] Birkenheuer A.J., Neel J., Ruslander D., Levy M.G., Breitschwerdt E.B., Detection and molecular characterization of a novel large Babesia species in a dog, Vet. Parasitol. (2004) 124:151-160.

[13] Blouin E.F., Van Rensburg L., An ultrastructural study of the development of Babesia occultans in the salivary glands of adult Hyalomma marginatum rufipes Onderstepoort, J. Vet. Res. (1988) 55:93-100.

[14] Bonnet S., Jouglin M., Malandrin L., Becker C., Agoulon A., L'hostis M., Chauvin A., Transstadial and transovarial persistence of Babesia divergens DNA in Ixodes ricinus ticks fed on infected blood in a new skin-feeding technique, Parasitology (2007) 134:197207.

[15] Botros B.A., Moch R.W., Barsoum I.S., Some observations on experimentally induced infection of dogs with Babesia gibsoni, Am. J. Vet. Res. (1975) 36:293-296.

[16] Brandao L.P., Hagiwara M.K., Myiashiro S.I., Humoral immunity and reinfection resistance in dogs experimentally inoculated with Babesia canis and either treated or untreated with imidocarb dipropionate, Vet. Parasitol. (2003) 114:253-265.

[17] Brayton K.A., Lau A.O., Herndon D.R., Hannick L., Kappmeyer L.S., Berens S.J., et al., Genome sequence of Babesia bovis and comparative analysis of apicomplexan hemoprotozoa, PLoS Pathog. (2007) 3:1401-1413.

[18] Brown W.C., Estes D.M., Chantler S.E., Kegerreis K.A., Suarez C.E., DNA and a CpG oligonucleotide derived from Babesia bovis are mitogenic for bovine B cells, Infect. Immun. (1998) 66:5423-5432.

[19] Brown W.C., Palmer G.H., Designing bloodstage vaccines against Babesia bovis and B. bigemina, Parasitol. Today (Regul. ed.) (1999) 15:275-281.

[20] Brown W.C., Molecular approaches to elucidating innate and acquired immune responses to Babesia bovis a protozoan parasite that causes persistent infection, Vet. Parasitol. (2001) 101:233-248.

[21] Brown W.C., Norimine J., Knowles D.P., Goff W.L., Immune control of Babesia bovis infection, Vet. Parasitol. (2006) 138:75-87.
[22] Callow L.L., Stewart N.P., Immunosuppression by Babesia bovis against its tick vector, Boophilus microplus, Nature (1978) 272:818-819.

[23] Callow L.L., Dalgliesh R.J., Immunity and immunopathology in babesiosis, in: Cohen S., Warren K.S. (Eds.), Immunology of parasitic infections, 2nd ed., Blackwell scientific publications, 1982, pp. 475526.

[24] Cantu A., Ortega-S J.A., Mosqueda J., GarciaVazquez Z., Henke S.E., George J.E., Immunologic and molecular identification of Babesia bovis and Babesia bigemina in free-ranging white-tailed deer in northern Mexico, J. Wildl. Dis. (2007) 43:504-507.

[25] Carcy B., Précigout E., Schetters T., Gorenflot A., Genetic basis for GPI-anchor merozoite surface antigen polymorphism of Babesia and resulting antigenic diversity, Vet. Parasitol. (2006) 138:33-49.

[26] Carret C., Walas F., Carcy B., Grande N., Précigout E., Moubri K., et al., Babesia canis canis, Babesia canis vogeli, Babesia canis rossi: differentiation of the three subspecies by a restriction fragment length polymorphism analysis on amplified small subunit ribosomal RNA genes, J. Eukaryot. Microbiol. (1999) 46:298-303.

[27] Cen-Aguilar J.F., Rodriguez-Vivas R.I., Dominguez-Alpizar J.L., Wagner G.G., Studies on the effect of infection by Babesia sp. on oviposition of Boophilus microplus engorged females naturally infected in the Mexican tropics, Vet. Parasitol. (1998) 78:253257.

[28] Chauvin A., Valentin A., Malandrin L., L'Hostis M., Sheep as a new experimental host for Babesia divergens, Vet. Res. (2002) 33:429-433.

[29] Christensson D.A., Inverse age resistance to experimental Babesia divergens infection in cattle, Acta Vet. Scand. (1989) 30:453-464.

[30] Concannon R., Wynn-Owen K., Simpson V.R., Birtles R.J., Molecular characterization of haemoparasites infecting bats (Microchiroptera) in Cornwall, UK, Parasitology (2005) 131:489-496.

[31] Criado A., Martinez J., Buling A., Barba J.C., Merino S., Jefferies R., Irwin P.J., New data on epizootiology and genetics of piroplasms based on sequences of small ribosomal subunit and cytochrome b genes, Vet. Parasitol. (2006) 142:238-247.

[32] Criado-Fornelio A., Martinez-Marcos A., Buling-Sarana A., Barba-Carretero J.C., Molecular studies on Babesia, Theileria and Hepatozoon in southern Europe. Part I. Epizootiological aspects, Vet. Parasitol. (2003) 113:189-201.

[33] Criado-Fornelio A., Martinez-Marcos A., Buling-Sarana A., Barba-Carretero J.C., Molecular studies on Babesia, Theileria and Hepatozoon in southern 
Europe. Part II. Phylogenetic analysis and evolutionary history, Vet. Parasitol. (2003) 114:173-194.

[34] Curnow J.A., Studies on antigenic changes and strain differences in Babesia argentina infections, Aust. Vet. J. (1973) 49:279-283.

[35] Dao A.H., Eberhard M.L., Pathology of acute fatal babesiosis in hamsters experimentally infected with the WA-1 strain of Babesia, Lab. Invest. (1996) 74:853-859.

[36] Davies S., Joyner L., Kendall S., Studies on Babesia divergens (M'Fadyean and Stockman, 1911), Ann. Trop. Med. Parasitol. (1958) 52:206-215.

[37] Dalgliesh R.J., Stewardt N.P., The extraction of infective Babesia bovis and Babesia bigemina from tick eggs and B. bigemina from unfed larval ticks, Aust. Vet. J. (1978) 54:453-454.

[38] de Vos A.J., Stewart N.P., Dalgliesh R.J., Effect of different methods of maintenance on the pathogenicity and infectivity of Babesia bigemina for the vector Boophilus microplus, Res. Vet. Sci. (1989) 46:139-142.

[39] Donnelly J., Peirce M.A., Experiments on the transmission of Babesia to cattle by the tick Ixodes ricinus, Int. J. Parasitol. (1975) 5:363-367.

[40] Enigk K., Friedhoff K., The host specificity of Babesia divergens (M'Fadyean and Stockman 1911), Zeitschr. Parasitenk. (1962) 21:238-256.

[41] Florin-Christensen J., Suarez C.E., Florin-Christensen M., Hines S.A., McElwain T.F., Palmer G.H., Phosphatidylcholine formation is the predominant lipid biosynthetic event in the hemoparasite Babesia bovis, Mol. Biochem. Parasitol. (2000) 106:147-156.

[42] Gaffar F.R., Franssen F.F., de Vries E., Babesia bovis merozoites invade human ovine, equine, porcine and caprine erythrocytes by a sialic acid-dependent mechanism followed by developmental arrest after a single round of cell fission, Int. J. Parasitol. (2003) 33:1595-1603.

[43] Garcia T.D., Figueroa M.J., Ramos A.J., Rojas M.C., Canto A.G., Falcon N.A., Alvarez M.J., Immune response to Babesia bigemina infection in pregnant cows, Ann. N.Y. Acad. Sci. (2004) 1026: $144-148$.

[44] Garnham P.C.C., Bray R.S., The susceptibility of the higher primates to piroplasms, J. Protozool. (1959) $6: 352-355$.

[45] Goff W.L., Wagner G.G., Craig T.M., Increased activity of bovine ADCC effector cells during acute Babesia bovis infection, Vet. Parasitol. (1984) 16:5-15.
[46] Goff W.L., Johnson W.C., Parish S.M., Barrington G.M., Tuo W., Valdez R.A., The age-related immunity in cattle to Babesia bovis infection involves the rapid induction of interleukin-12, interferongamma and inducible nitric oxide synthase mRNA expression in the spleen, Parasite Immunol. (2001) 23:463-471.

[47] Goff W.L., Johnson W.C., Parish S.M., Barrington G.M., Elsasser T.H., Davis W.C., Valdez R.A., IL-4 and IL-10 inhibition of IFN-gamma- and TNF-alpha-dependent nitric oxide production from bovine mononuclear phagocytes exposed to Babesia bovis merozoites, Vet. Immunol. Immunopathol. (2002) 84: 237-251

[48] Goff W.L., Storset A.K., Johnson W.C., Brown W.C., Bovine splenic NK cells synthesize IFN-gamma in response to IL-12-containing supernatants from Babesia bovis-exposed monocyte cultures, Parasite Immunol. (2006) 28:221-228.

[49] Gordon J.L., Sibley L.D., Comparative genome analysis reveals a conserved family of actin-like proteins in apicomplexan parasites, BMC Genomics (2005) 6:179.

[50] Gorenflot A., Brasseur P., Precigout E., L'hostis M., Marchand A., Schrevel J., Cytological and immunological responses to Babesia divergens in different hosts: ox, gerbil, man, Parasitol. Res. (1991) 77: 3-12.

[51] Gough J.M., Jorgensen W.K., Kemp D.H., Development of tick gut forms of Babesia bigemina in vitro, J. Eukaryot. Microbiol. (1998) 45:298-306.

[52] Gray J.S., The effects of the piroplasm Babesia bigemina on the survival and reproduction of the blue tick, Boophilus decoloratus, J. Invertebr. Pathol. (1982) 39:413-415.

[53] Gray J.S., Langley R.J., Murphy T.M., Morphological comparisons of the bovine piroplasm, Babesia divergens, in cattle and jird (Meriones unguiculatus) erythrocytes, J. Parasitol. (1985) 71:799-802.

[54] Guan G.Q., Yin H., Luo J.X., Lu W.S., Zhang Q.C., Gao Y.L., Lu B.Y., Transmission of Babesia sp. to sheep with field-collected Haemaphysalis qinghaiensis, Parasitol. Res. (2002) 88:S22-S24.

[55] Habela M., Reina D., Nieto C., Navarrete I., Antibody response and duration of latent infection in sheep following experimental infection with Babesia ovis, Vet. Parasitol. (1990) 35:1-10.

[56] Hadj-Kaddour K., Carcy B., Vallet A., Randazzo S., Delbecq S., Kleuskens J., et al., Recombinant protein $\mathrm{Bd} 37$ protected gerbils against heterologous challenges with isolates of Babesia divergens polymorphic for the bd37 gene, Parasitology (2007) 134:187-196. 
[57] Herwaldt B.L., Caccio S., Gherlinzoni F., Aspöck H., Slemenda S.B., Piccaluga P., et al., Molecular characterization of a non-Babesia divergens organism causing zoonotic babesiosis in Europe, Emerg. Infect. Dis. (2003) 9:942-948.

[58] Higuchi S., Oya H., Hoshi F., Kawamura S., Yasuda Y., Development of Babesia ovata in the salivary glands of the nymphal tick Haemaphysalis longicornis, J. Vet. Med. Sci. (1994) 56:207-209.

[59] Higuchi S., Fujimori M., Hoshi F., Kawamura S., Yasuda Y., Development of Babesia gibsoni in the salivary glands of the larval tick Rhipicephalus sanguineus, J. Vet. Med. Sci. (1995) 57:117-119.

[60] Holman P.J., Spencer A.M., Droleskey R.E., Goethert H.K., Telford S.R., In vitro cultivation of a zoonotic Babesia sp. isolated from eastern cottontail rabbits (Sylvilagus floridanus) on Nantucket Island, Massachusetts, J. Clin. Microbiol. (2005) 43:39954001.

[61] Howell J.M., Ueti M.W., Palmer G.H., Scoles G.A., Knowles D.P., Persistently infected calves as reservoirs for acquisition and transovarial transmission of Babesia bovis by Rhipicephalus (Boophilus) microplus, J. Clin. Microbiol. (2007) 45:3155-3159.

[62] Hunfeld K., Hildebrandt A., Gray J., Babesiosis: Recent insights into an ancient disease, Int. J. Parasitol. (2008) 38:1219-1237.

[63] Jaworski D.C., Jasinskas A., Metz C.N., Bucala R., Barbour A.G., Identification and characterization of a homologue of the pro-inflammatory cytokine Macrophage Migration Inhibitory Factor in the tick, Amblyomma americanum, Insect Mol. Biol. (2001) 10:323-331.

[64] Joyner L.P., Davies S.F., Kendall S.B., The experimental transmission of Babesia divergens by Ixodes ricinus, Exp. Parasitol. (1963) 14:367-373.

[65] Langley R.J., Gray J.S., Age-related susceptibility of the gerbil, Meriones unguiculatus, to the bovine parasite, Babesia divergens, Exp. Parasitol. (1987) 64:466-473.

[66] Latif B., Said M., Ali S., Effect of age on the immune response of cattle experimentally infected with Babesia bigemina, Vet. Parasitol. (1979) 5:307-314.

[67] Leander B.S., Marine gregarines: evolutionary prelude to the apicomplexan radiation?, Trends Parasitol. (2008) 24:60-67.

[68] Leroith T., Brayton K.A., Molloy J.B., Bock R.E., Hines S.A., Lew A.E., McElwain T.F., Sequence variation and immunologic cross-reactivity among Babesia bovis merozoite surface antigen 1 proteins from vaccine strains and vaccine breakthrough isolates, Infect. Immun. (2005) 73:5388-5394.
[69] Lewis D., Williams H., Infection of the Mongolian gerbil with the cattle piroplasm Babesia divergens, Nature (1979) 278:170-171.

[70] Liddell K.G., Joss A.W., Williams H., Serological response of the Mongolian gerbil to Babesia divergens (human strain) infection, Ann. Trop. Med. Parasitol. (1982) 76:527-538.

[71] Lipsitch M., Nowak M.A., Ebert D., May R.M., The population dynamics of vertically and horizontally transmitted parasites, Proc. Biol. Sci. (1995) 260: 321-327.

[72] Liu A.H., Yin H., Guan G.Q., Schnittger L., Liu Z.J., Ma M.L., et al., At least two genetically distinct large Babesia species infective to sheep and goats in China, Vet. Parasitol. (2007) 147:246-251.

[73] Mackenstedt U., Gauer M., Mehlhorn H., Schein E., Hauschild S., Sexual cycle of Babesia divergens confirmed by DNA measurements, Parasitol. Res. (1990) 76:199-206.

[74] Mackenstedt U., Gauer M., Fuchs P., Zapf F., Schein E., Mehlhorn H., DNA measurements reveal differences in the life cycles of Babesia bigemina and B. canis, two typical members of the genus Babesia, Parasitol. Res. (1995) 81:595-604.

[75] Mahoney D.F., Wright I.G., Mirre G.B., Bovine babesiasis: the persistence of immunity to Babesia argentina and B. bigemina in calves (Bos taurus) after naturally acquired infection, Ann. Trop. Med. Parasitol. (1973) 67:197-203.

[76] Malandrin L., L'Hostis M., Chauvin A., Isolation of Babesia divergens from carrier cattle blood using in vitro culture, Vet. Res. (2004) 35:131-139.

[77] Malandrin L., Jouglin M., Moreau E., Chauvin A., Individual heterogeneity in erythrocyte susceptibility to Babesia divergens is a critical factor for the outcome of experimental spleen-intact sheep infections, Vet. Res. (2009) 40:25.

[78] Martinod S., Brossard M., Moreau Y., Immunity of dogs against Babesia canis, its vector tick Dermacentor reticulatus, and Ixodes ricinus in endemic area, J. Parasitol. (1985) 71:269-273.

[79] Martinod S., Laurent N., Moreau Y., Resistance and immunity of dogs against Babesia canis in an endemic area, Vet. Parasitol. (1986) 19:245-254.

[80] Martinsen E., Perkins S., Schall J., A threegenome phylogeny of malaria parasites (Plasmodium and closely related genera): Evolution of life-history traits and host switches, Mol. Phylogenet. Evol. (2008) 47:261-273.

[81] Mehlhorn H., Schein E., The piroplasms: life cycle and sexual stages, Adv. Parasitol. (1984) 23: $37-103$. 
[82] Mehlhorn H., Parasitology in Focus: Facts and Trends, Springer, Berlin 1988.

[83] Menge D.M., Zhong D., Guda T., Gouagna L., Githure J., Beier J., Yan G., Quantitative trait loci controlling refractoriness to Plasmodium falciparum in natural Anopheles gambiae mosquitoes from a malaria-endemic region in western Kenya, Genetics (2006) 173:235-241.

[84] Moltmann U.G., Mehlhorn H., Friedhoff K.T., Electron microscopic study on the development of Babesia ovis (Piroplasmia) in the salivary glands of the vector tick Rhipicephalus bursa, Acta Trop. (1982) 39:29-40.

[85] Mosqueda J., McElwain T.F., Palmer G.H., Babesia bovis merozoite surface antigen 2 proteins are expressed on the merozoite and sporozoite surface, and specific antibodies inhibit attachment and invasion of erythrocytes, Infect. Immun. (2002) 70:6448-6455.

[86] Mosqueda J., McElwain T.F., Stiller D., Palmer G.H., Babesia bovis merozoite surface antigen 1 and rhoptry-associated protein 1 are expressed in sporozoites, and specific antibodies inhibit sporozoite attachment to erythrocytes, Infect. Immun. (2002) 70:1599-1603.

[87] Mosqueda J., Falcon A., Antonio Alvarez J., Alberto Ramos J., Oropeza-Hernandez L.F., Figueroa J.V., Babesia bigemina sexual stages are induced in vitro and are specifically recognized by antibodies in the midgut of infected Boophilus microplus ticks, Int. J. Parasitol. (2004) 34:1229-1236.

[88] Nilsson O., Nordkvist M., Rydén L., Experimental Babesia divergens infection in reindeer (Rangifer tarandus), Acta Vet. Scand. (1965) 6:353-359.

[89] O'Connor R.M., Allred D.R., Selection of Babesia bovis-infected erythrocytes for adhesion to endothelial cells coselects for altered variant erythrocyte surface antigen isoforms, J. Immunol. (2000) 164:2037-2045.

[90] O’Donoghue P.J., Friedhoff K.T., Vizcaino O.G., Weyreter H., The detection of IgM and IgG antibodies against Babesia bigemina in bovine sera using semidefined antigens in enzyme immunoassays, Vet. Parasitol. (1985) 18:1-12.

[91] Ouhelli H., Schein E., Effect of temperature on transovarial transmission of Babesia bigemina (Smith and Kilborne, 1893) in Boophilus annulatus (Say, 1821), Vet. Parasitol. (1988) 26:229-235.

[92] Pagel Van Zee J., Geraci N.S., Guerrero F.D., Wikel S.K., Stuart J.J., Nene V.M., Hill C.A., Tick genomics: the Ixodes genome project and beyond, Int. J. Parasitol. (2007) 37:1297-1305.

[93] Peirce M.A., A taxonomic review of avian piroplasms of the genus Babesia Starcovici, 1893
(Apicomplexa: Piroplasmorida: Babesiidae), J. Nat. Hist. (2000) 34:317-332.

[94] Penzhorn B.L., Babesiosis of wild carnivores and ungulates, Vet. Parasitol. (2006) 138:11-21.

[95] Phillips R.S., Babesia divergens in splenectomised rats, Res. Vet. Sci. (1984) 36:251-255.

[96] Potgieter F., Els H., Light and electron microscopic observations on the development of small merozoites of Babesia bovis in Boophilus microplus larvae, Onderstepoort J. Vet. Res. (1976) 43:123-128.

[97] Precigout E., Delbecq S., Vallet A., Carcy B., Camillieri S., Hadj-Kaddour K., et al., Association between sequence polymorphism in an epitope of Babesia divergens $\mathrm{Bd} 37$ exoantigen and protection induced by passive transfer, Int. J. Parasitol. (2004) 34:585-593.

[98] Rachinsky A., Guerrero F.D., Scoles G.A., Differential protein expression in ovaries of uninfected and Babesia-infected southern cattle ticks, Rhipicephalus (Boophilus) microplus, Insect. Biochem. Mol. Biol. (2007) 37:1291-1308.

[99] Rachinsky A., Guerrero F.D., Scoles G.A., Proteomic profiling of Rhipicephalus (Boophilus) microplus midgut responses to infection with Babesia bovis, Vet. Parasitol. (2008) 152:294-313.

[100] Randolph S.E., Fauna, climate and politics: possible causes for the recent increases in tick-borne zoonoses, Arch. Pédiatr. (2004) 11:1282-1285 (in French).

[101] Romany M.M., Yousef M.R., Roshdy O.H., Daoud A.M., Immunosuppressive effect of Babesia bovis immunogens on immunization against foot and mouth disease, Vet. Med. J. Giza (2006) 54:611-621.

[102] Rudenko N., Golovchenko M., Grubhoffer L., Gene organization of a novel defensin of Ixodes ricinus: first annotation of an intron/exon structure in a hard tick defensin gene and first evidence of the occurrence of two isoforms of one member of the arthropod defensin family, Insect. Mol. Biol. (2007) 16:501-507.

[103] Rudzinska M.A., Spielman A., Riek R.F., Lewengrub S.J., Piesman J., Intraerythrocytic "gametocytes" of Babesia microti and their maturation in ticks, Can. J. Zool. (1979) 57:424-434.

[104] Rudzinska M.A., Spielman A., Lewengrub S., Trager W., Piesman J., Sexuality in piroplasms as revealed by electron microscopy in Babesia microti, Proc. Natl. Acad. Sci. USA (1983) 80:2966-2970.

[105] Schein E., Mehlhorn H., Voigt W.P., Electron microscopical studies on the development of Babesia canis (Sporozoa) in the salivary glands of the vector tick Dermacentor reticulatus, Acta Trop. (1979) 36:229-241. 
[106] Schetters T.P., Kleuskens J., Scholtes N., Gorenflot A., Parasite localization and dissemination in the Babesia-infected host, Ann. Trop. Med. Parasitol. (1998) 92:513-519.

[107] Schnittger L., Yin H., Gubbels M.J., Beyer D., Niemann S., Jongejan F., Ahmed J.S., Phylogeny of sheep and goat Theileria and Babesia parasites, Parasitol. Res. (2003) 91:398-406.

[108] Shoda L.K., Palmer G.H., Florin-Christensen J., Florin-Christensen M., Godson D.L., Brown W.C., Babesia bovis-stimulated macrophages express interleukin-1beta, interleukin-12, tumor necrosis factor alpha, and nitric oxide and inhibit parasite replication in vitro, Infect. Immun. (2000) 68:5139-5145.

[109] Shoda L.K., Kegerreis K.A., Suarez C.E., Roditi I., Corral R.S., Bertot G.M., et al., DNA from protozoan parasites Babesia bovis, Trypanosoma cruzi, and T. brucei is mitogenic for B lymphocytes and stimulates macrophage expression of interleukin12, tumor necrosis factor alpha, and nitric oxide, Infect. Immun. (2001) 69:2162-2171.

[110] Sojka D., Franta Z., Horn M., Hajdusek O., Caffrey C.R., Mares M., Kopcek P., Profiling of proteolytic enzymes in the gut of the tick Ixodes ricinus reveals an evolutionarily conserved network of aspartic and cysteine peptidases, Parasit. Vectors (2008) 1:7.

[111] Sreter T., Szeli Z., Varga I., Spatial distribution of Dermacentor reticulatus and Ixodes ricinus in Hungary: evidence for change?, Vet. Parasitol. (2005) 128:347-351.

[112] Steen N.A., Barker S.C., Alewood P.F., Proteins in the saliva of the Ixodida (ticks): pharmacological features and biological significance, Toxicon (2006) 47:1-20.

[113] Stich R.W., Shoda L.K., Dreewes M., Adler B., Jungi T.W., Brown W.C., Stimulation of nitric oxide production in macrophages by Babesia bovis, Infect. Immun. (1998) 66:4130-4136.

[114] Suarez C.E., Florin-Christensen M., Hines S.A., Palmer G.H., Brown W.C., McElwain T.F., Characterization of allelic variation in the Babesia bovis merozoite surface antigen 1 (MSA-1) locus and identification of a cross-reactive inhibition-sensitive MSA-1 epitope, Infect. Immun. (2000) 68:6865-6870.

[115] Talman A., Domarle O., Mckenzie F., Ariey F., Robert V., Gametocytogenesis: the puberty of Plasmodium falciparum, Malar. J. (2004) 3-24.

[116] Trees A.J., Indirect fluorescent antibody levels in experimental Babesia divergens infections of cattle, Res. Vet. Sci. (1978) 24:126-128.

[117] Trueman K.F., Blight G.W., The effect of age on resistance of cattle to Babesia bovis, Aust. Vet. J. (1978) 54:301-305. [117b] Tsao J.I., Reviewing molecular adaptations of Lyme borreliosis spirochetes in the context of reproductive fitness in natural transmission cycles, Vet. Res. (2009) 40:36.

[118] Tsuji N., Battsetseg B., Boldbaatar D., Miyoshi T., Xuan X., Oliver J.H., Fujisaki K., Babesial vector tick defensin against Babesia sp. parasites, Infect. Immun. (2007) 75:3633-3640.

[119] Tsuji N., Miyoshi T., Battsetseg B., Matsuo T., Xuan X., Fujisaki K., A cysteine protease is critical for Babesia spp. transmission in Haemaphysalis ticks, PLoS Pathog. (2008) 4:e1000062.

[120] Uilenberg G., Babesia - a historical overview, Vet. Parasitol. (2006) 138:3-10.

[121] Umemiya R., Hatta T., Liao M., Tanaka M., Zhou J., Inoue N., Fujisaki K., Haemaphysalis longicornis: molecular characterization of a homologue of the macrophage migration inhibitory factor from the partially fed ticks, Exp. Parasitol. (2007) 115:137-142.

[122] Wlosniewski A., Leriche M.A., Chavigny C., Ulmer P., Donnay V., Boulouis H.J., et al., Asymptomatic carriers of Babesia canis in an enzootic area, Comp. Immunol. Microbiol. Infect. Dis. (1997) 20:75-86.

[123] Yeruham I., Hadani A., Galker F., The effect of the ovine host parasitaemia on the development of Babesia ovis (Babes, 1892) in the tick Rhipicephalus bursa (Canestrini and Fanzago, 1877), Vet. Parasitol (2001) 96:195-202.

[124] Yokoyama N., Suthisak B., Hirata H., Matsuo T., Inoue N., Sugimoto C., Igarashi I., Cellular localization of Babesia bovis merozoite rhoptry-associated protein 1 and its erythrocyte-binding activity, Infect. Immun. (2002) 70:5822-5826.

[125] Young A.S., Morzaria S.P., Biology of Babesia, Parasitol. Today (Regul. ed.) (1986) 2:211-219.

[126] Zahler M., Schein E., Rinder H., Gothe R., Characteristic genotypes discriminate between Babesia canis isolates of differing vector specificity and pathogenicity to dogs, Parasitol. Res. (1998) 84:544-548.

[127] Zintl A., Westbrook C., Skerrett H.E., Gray J.S., Mulcahy G., Chymotrypsin and neuraminidase treatment inhibits host cell invasion by Babesia divergens (Phylum Apicomplexa), Parasitology (2002) 125: 45-50.

[128] Zintl A., Mulcahy G., Skerrett H.E., Taylor S.M., Gray J.S., Babesia divergens, a bovine blood parasite of veterinary and zoonotic importance, Clin. Microbiol. Rev. (2003) 16:622-636.

[129] Zintl A., Gray J.S., Skerrett H.E., Mulcahy G., Possible mechanisms underlying age-related resistance to bovine babesiosis, Parasite Immunol. (2005) 27: 115-120. 